\title{
Use of amiloride in lithium-induced nephrogenic diabetes insipidus
}

\begin{abstract}
Lithium is the main cause of secondary nephrogenic diabetes insipidus, occurring in approximately $20 \%$ of patients that use this drug chronically. Amiloride has been proposed as an alternative for those patients who develop lithium-induced nephrogenic diabetes insipidus in whom drug suspension is not the best option. This drug prevents the entry of lithium to the nephron by blocking the epithelial sodium channel $(\mathrm{ENaC})$ in the main cells. The suggested doses are $5 \mathrm{mg}$ twice a day, and can be combined with hydrochlorothiazide at a dose of $50 \mathrm{mg}$ per day. Numerous studies have demonstrated the beneficial effect of amiloride on lithium-induced nephrogenic diabetes insipidus, however, its long-term safety and effectiveness have not been evaluated.
\end{abstract}

Volume 7 Issue 3 - 2018

\section{Danilo Cardona R}

Pregrado en medicina, Universidad de Caldas, Colombia

Correspondence: Danilo Cardona R, Facultad de Ciencias Para la Salud, Universidad de Caldas, Av. Paralela No. 48-57-Manizales, Colombia, Tel +57-32I75I4775, Email jhojan.521214567@ucaldas.edu.co

Received: April 19, 2018 | Published: June 06, 2018

Keywords: nephrogenic diabetes insipidus, lithium, amiloride, $\mathrm{ENaC}$

\section{Introduction}

Lithium is a medication widely used in psychiatry for the management of bipolar spectrum disorders. ${ }^{1}$ About half of the patients who receive this drug have some degree of impaired renal urinary concentration capacity and about $20 \%$ develop symptomatic nephrogenic diabetes insipidus. ${ }^{1,2}$ This entity is characterized by excretion of large volumes of poorly concentrated urine and lithium is the main cause of secondary nephrogenic diabetes insipidus. ${ }^{1,3}$ This medicine can also cause fibrosis and kidney failure, with an increase in the incidence of adverse reactions associated with the use of lithium since it is purified primarily through the urine. ${ }^{4}$

Lithium is a metal that behaves as a monovalent cation, sharing characteristics with sodium and potassium, enters the nephron through the epithelial sodium channel $(\mathrm{ENaC})$ and accumulates in this organ, causing downregulation of the aquaporin 2 and Urea transporters (UTA1 and UT-B), injuring the main cells of the collecting tubule in the nephron, which impairs the renal capacity to concentrate the urine. ${ }^{3,5}$

Despite the severity of adverse reactions, there are patients in whom the suspension of lithium could be counterproductive, such as those who have only improved their symptoms with the continued use of this medication and those with increased suicidal ideation after suspend it, for which strategies have been sought to prevent the onset or attenuate the severity of nephrogenic diabetes insipidus induced by lithium. ${ }^{3}$ Amiloride is currently a possible option with promising results in this disorder. ${ }^{6,7}$

\section{Discussion}

The mechanism by which amiloride antagonizes the nephrotoxic effects of lithium, is mainly determined by the inhibition of the epithelial sodium channel in the main cells of the nephron, which prevents the entrance of this metal to the renal cells and the downregulation in the Aquaporin 2 expression, and therefore the disorder in the concentration of urine. ${ }^{1,7-9}$ However, the mechanism by which it has tried to explain the prevention of the progression of renal fibrosis induced by lithium is the decrease in the expression of the profibrotic cytokine, which decreases the activity of the myofibroblasts and the collagen deposition in the kidney.
Kortenoeven et al., ${ }^{7}$ showed in a murine renal cell model a decrease in transcellular transport and the renal concentration of lithium, with the use of amiloride, which produced a decrease in down regulation of aquaporin 2, with improvement of polyuria and an increase in urinary osmolarity. In this study, although a reduction in the expression of the glycogen synthase kinase $3 \mathrm{~b}$ was not demonstrated with the use of amiloride, an effect was demonstrated on the phosphorylation of this enzyme to Ser9, another mechanism involved in the down regulation of the Aquaporin 2 induced by lithium. $3,7,10$

Other studies in animal models, such as that of Kalita-De et al., 5 have also shown the beneficial effects of amiloride on the progression of kidney damage associated with the chronic use of lithium and therefore the development of secondary nephrogenic diabetes insipidus, explaining this effect by mechanisms similar to those described by Kortenoeven et al. ${ }^{7}$ However, there are few studies that demonstrate this effect in humans and there are no studies that evaluate the long-term impact of amiloride in this condition, so that it can be established if it stops progression of the established lesion, reverses it or prevent it. ${ }^{6,11}$ Despite this, some authors suggest a dose of amiloride of $5 \mathrm{mg}$ twice a day as effective to prevent lithium-induced polyuria, although there are no recommendations on the ideal time to start this medication or the patients who are candidates for its use. ${ }^{7}$ Doses of $0.3 \mathrm{mg} / \mathrm{kg} /$ day have been reported in pediatric patients. ${ }^{3,8}$

Some studies have described the use of amiloride in combination with hydrochlorothiazide, ${ }^{1}$ although this combination may result in an increase in lithium-induced toxicity, since hydrochlorothiazide increases the serum levels of this drug, requiring a reduction of the dose to one third of usual dose of lithium, which implies an additional risk of adverse effects. ${ }^{10}$ This combination can also cause hypokalemia and other hydroelectrolyte alterations associated with the use of thiazide diuretics. ${ }^{10}$ However, some authors recommend this combination as the first choice, at doses of $5 \mathrm{mg}$ of amiloride 2 times a day and 50mg of hydrocorothiazide daily.

A limitation of the studies that evaluate the ability of amiloride to avoid renal fibrosis and nephrogenic diabetes insipidus induced by lithium is the early start of this drug (in the first 4 weeks of the start of lithium therapy), since in the Clinical practice shows that patients who have more adverse reactions with the use of lithium have been using 
it for months or years, which hypothetically would lead to greater renal damage at the time of initiating amiloride compared to the study subjects. ${ }^{5,12}$ On the other hand, this could suggest that the start of amiloride should be carried out in the early stages of lithium therapy, seeking a greater protective effect when impacting in the early stages of kidney injury or before it takes place.

\section{Conclusion}

The different mechanisms by which amiloride prevents nephrogenic diabetes insipidus induced by lithium have been widely demonstrated, the most prominent being the inhibition of the epithelial sodium channel in the apical membrane of the main cells. A dose of $5 \mathrm{mg}$ twice daily has been suggested in patients with polyuria associated with the chronic use of lithium. However, there are no studies evaluating the long-term impact of this drug on lithiuminduced kidney damage or the patients who would benefit most from its use, being an area for future research.

\section{Acknowledgements}

None.

\section{Conflict of interest}

The author declares that there is no conflict of interest.

\section{References}

1. Gitlin M. Lithium side effects and toxicity: prevalence and management strategies Background. Int J Bipolar Disord. 2016;4(1):27.

2. Rej S, Herrmann N, Shulman K. The Effects of Lithium on Renal Function in Older Adults-A Systematic Review. J Geriatr Psychiatry Neurol. 2012;25(1):51-61.
3. Velásquez Jones L, Medeiros Domingo M. Diabetes insípida nefrogénica. Boletín Médico del Hospital Infantil de México. 2014;71(6):332-338.

4. Félix P, Stoermann-Chopard C, Martin PY. Lithium and chronic kidney disease: a pathology which remains relevant. Rev Med Suisse. 2010;6(238):448-452.

5. Kalita-De Croft P, Bedford JJ, Leader JP, et al. Amiloride modifies the progression of lithium-induced renal interstitial fibrosis. Nephrology. 2018;23(1):20-30.

6. Pattanayak RD, Rajhans P, Shakya P, et al. Lithium-induced polyuria and amiloride: Key issues and considerations. Indian J Psychiatry. 2017;59(3):391-392.

7. Kortenoeven MLA, Li Y, Shaw S, et al. Amiloride blocks lithium entry through the sodium channel thereby attenuating the resultant nephrogenic diabetes insipidus. Kidney Int. 2009;76(1):44-53.

8. Wesche D, Deen PMT, Knoers NVAM. Congenital nephrogenic diabetes insipidus: the current state of affairs. Pediatr Nephrol. 2012;27(12):21832204.

9. Bedford JJ, Weggery S, Ellis G, et al. Lithium-induced Nephrogenic Diabetes Insipidus: Renal Effects of Amiloride. Clin J Am Soc Nephrol. 2008;3(5):1324-1331.

10. Sinke AP, Kortenoeven MLA, de Groot T, et al. Hydrochlorothiazide attenuates lithium-induced nephrogenic diabetes insipidus independently of the sodium-chloride cotransporter. Am $J$ Physiol Physiol. 2014;306(5):F525-F533.

11. Finch CK, Kelley KW, Williams RB. Treatment of lithium-induced diabetes insipidus with amiloride. Pharmacotherapy. 2003;23(4):546550 .

12. Rybakowski J, Drogowska J, Abramowicz M, et al. The effect of longterm lithium treatment on kidney function. Psychiatr Pol. 46(4):627-636. 